\title{
Biofilm Formation in Multi-Drug Resistant Staphylococcus Aureus Isolates from Hospitalized Patients
}

\author{
Abdolmajid Ghasemian ${ }^{1}$, Shahin Najar Peerayeh ${ }^{2}$, Bita Bakhshi $^{3}$, Mohsen Mirzaee $^{4}$
}

${ }^{1}$ Ph.D. student, Department of Bacteriology, Faculty of Medical Sciences, Tarbiat Modares University, Tehran, Iran.; ${ }^{2}$ Associate Professor, Department of Bacteriology, Faculty of Medical Sciences, Tarbiat Modares University, Tehran, Iran; ${ }^{3}$ Assistant Professor, Department of Bacteriology, Faculty of Medical Sciences, Tarbiat Modares University, Tehran, Iran; ${ }^{3}$ Assistant Professor, Faculty of Laboratory Sciences, Borujerd Branch, Islamic Azad University, Borujerd, Iran

[Received: 1 January 2014; Accepted: 15 March 2014; Published: 1 December 2014]

\begin{abstract}
Background: The biofilm production is an important phenomenon by bacteria such as Staphylococcus aureus that contribute to the multiple drug resistance. Moreover, biofilm formation by multidrug-resistant Staphylococcus aureus causes evading from immune responses. Objective: The aim of this study was to detect biofilm formation and presence of several related genes among multidrug-resistant (MDR) isolates of Staphylococcus aureus. Methods: This cross sectional study was conducted at a hospital in Tehran, Iran from July 2012 to January 2013. Patients admitted with the infections of the different sites of the body were selected as study population. Staphylococcus aureus isolates were collected from hospitalized patients and identified by conventional diagnostic tests. The multidrug-resistant MRSA isolates were detected by antibiotic susceptibility test. The phenotypic biofilm formation was detected by micro-titre tissue plate assay. The polymerase chain reaction (PCR) was performed to detect the mecA, Staphylococcal Cassette Chromosome mec (SCCmec) types, accessory gene regulatory (agr) genes, the icaADBC and several genes encoding staphylococcal surface proteins including $c l f A B, f n b A B$, fib, eno, can, ebps and $b b p$ genes with specific primers. Results: A total number of 209 Staphylococcus aureus were isolated of which 64(30.6\%) isolates were methicillin-resistant; among which 36(56.2\%) isolates were MDR. These isolates were resistant to amoxicillin, tetracycline, ciprofloxacin, gentamicin, erythromycin and trimethoprim-sulfamethoxazole. All the isolates were susceptible to vancomycin and linezolid. All the MDR-MRSA harbored SCCmec type III. All the MDR- MRSA isolates were strong biofilm producers in the phenotypic test. Conclusions: Multidrug-resistant MRSA isolates produced biofilm strongly and the majority of these isolates harbored most of biofilm related genes.[Bangladesh J Infect Dis 2014;1(2):27-31]
\end{abstract}

Keywords: Staphylococcus aureus, MRSA, Multidrug-resistance, biofilm formation

Corresponding author: Dr. Shahin Najar Peerayeh, Department of Bacteriology, Faculty of Medical Sciences, Tarbiat Modares University, Tehran, Iran. Tel: +98-92182883870, Email: najarp_s@ @modares.ac.ir

[How to Cite this article: Ghasemian A, Peerayeh SN, Bakhshi B, Mirzaee M. Biofilm Formation in Multi-Drug Resistant Staphylococcus Aureus Isolates From Hospitalized Patients. Bangladesh J Infect Dis 2014;1(2):27-31]

Conflict of Interest: The authors declared no conflict of interest.

Contributions to authors: AG performed the laboratory work; NP designed the study; BB advised this work; MM helped the laboratory work. AG performed the clinical experiment; NP advised the work; BB and MM provided purely technical help, copyediting, proofreading or translation assistance

Funding agency: This work was supported by grants from Faculty of Medical Sciences, Tarbiat Modares University, Tehran, Iran. This survey was supported by grants from Tarbiat Modares University. Loghman Hospital and Tarbiat Modares University provided the general support. 


\section{Introduction}

The development of multidrug resistance by Staphylococcus aureus (MDR SA) is a public health concern especially due to MRSA isolates in nosocomial settings. In the hospital milieu, infected and colonized patients contribute to the transmission and spreading of $S$. aureus and hospital personnel, serving as reservoirs, facilitate further dissemination ${ }^{1-}$ ${ }^{2}$. Infections caused by MRSA often prove difficult to treat because of high levels of resistance to multiple antibiotics as a result of both intrinsic and acquired mechanisms ${ }^{3}$. Moreover, in recent years vancomycin resistance have made treatment of these isolates very difficult ${ }^{4}$. Drug resistance in $S$. aureus is occurred by complex genetic arrays such as Staphylococcal cassette chromosome mec (SCCmec) elements for methicillin-resistance and likewise all the beta-lactam antibiotics via production of new Penicillin Binding Protein2a [PBP2a $]^{5}$. MRSA isolates that are acquired from nosocomial milieu are referred as Healthcare Associated (HA)-MRSA. Various nosocomial infections such as those associated with the use of central venous catheters, prosthetic heart valves, urinary catheters and orthopedics devices contribute to the biofilm formation and persistent infections that culminate in evading immune system responses and antibiotic resistance ${ }^{6}$. Susceptibility tests with invitro biofilm models have depicted the survival of bacteria in biofilms after treatment with antibiotics with even much more than the minimum inhibitory concentrations. Moreover, in the body, chemotherapy dose not kill bacteria in biofilms, and when stopped, culminates in growing and spreading of the bacteria from biofilms ${ }^{7}$. Biofilm formation also mediates the spreading of the antibiotic resistance traits in nosocomial pathogens by developing mutation rates and the exchange of genes responsible for antibiotic resistance $^{8}$. Biofilms are closely involved in higher antibiotic resistance because of several conditions including lower penetration of antibiotics, lower growth rate of bacteria in biofilms and altered metabolic requirements. Eventually, the surviving isolates in the inner portion of biofilms are likely to possess a higher probability of acquiring the ability to develop biofilms as well as multidrug resistance in clinical settings ${ }^{9}$. Biofilm forming strains are more frequently isolated from non-fluid tissues, in particular bone and soft tissues, and also MDR pathogens are more often biofilm formers. Several studies have shown that methicillin-resistance can alter the ability of the $S$. aureus isolates regarding biofilm production ${ }^{10}$. Previous studies on the biofilm formation among MDR isolates and the relationship to clinical manifestations are scarce ${ }^{11}$. The aim of this study was to detect the biofilm production and the biofilm encoding genes in MDR S. aureus isolates.

\section{Methodology}

This cross sectional study was conducted at a hospital in Tehran, Iran from July 2012 to January 2013. Patients admitted with the infections of the different sites of the body were selected as study population. The $S$. aureus isolates were selected from a hospital. MDR SA isolates were collected from different clinical sites of the patients and the specimens were blood, tracheal aspirate, wound swab, sputum and other. The isolates were collected from intensive care unit (ICU), infectious diseases, in-patient and pediatrics settings. In addition, the isolates were identified with catalase, coagulases, acid production from mannitol on mannitol salt agar and DNase tests. The antibiotic susceptibility pattern of $S$. aureus isolates was drawn according to Clinical and Laboratory Standards Institute (CLSI, Kirby Bauer assay) that detected MDR- MRSA isolates. The phenotypic detection of MRSA was conducted with oxacillin $(1 \mu \mathrm{g})$ disk (MAST, UK). Total genomic DNA was extracted by preparation of a suspension of bacterial isolates in $200 \mu \mathrm{l}$ of TE buffer and

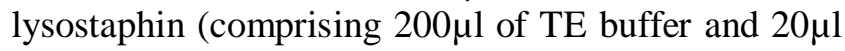
of lysostaphin $[2 \mu \mathrm{g} / \mathrm{ml}$, Sigma]). The DNA was isolated according to Straubinger method ${ }^{12}$. The DNA was amplified with specific primers to detect the $m e c A$ gene, SCCmec types, agr specific groups and biofilm related genes, including the icaADBC, clfAB, $f n b A B$, cna, ebps, eno, fib and bbp genes among the clinical isolates. The annealing temperature was $55^{\circ} \mathrm{C}$ (30s) for $m e c A$ gene and $51^{\circ} \mathrm{C}(1 \mathrm{~min})$ for SCCmec types, according to Zhang study ${ }^{13}$. For observation of the PCR products by electrophoresis, $5 \mu 1$ of each product was mixed with $1 \mu \mathrm{l}$ of each gel red and loading buffer dyes, and were run in $1 \%$ agarose gel electrophoresis and was observed by transluminator uv. The relationship between multidrug resistance and biofilm formation was evaluated by the Pearson ChiSquare test where any difference less than 0.05 was considered significant.

\section{Results}

The bacterial isolates were collected from different hospital settings and clinical sites, according to tubular forms 1 and 2. Among the total of $209 S$. aureus isolates, $30.6 \%(\mathrm{n}=64)$ were methicillinresistant with oxacillin disk and in addition by detection of mecA gene with $147 \mathrm{bp}$ size. Of 64 MRSA, 56\% ( $\mathrm{n}=36)$ were resistant to all the used antibiotics (MDR MRSA) including amoxicillin, 
tetracycline, erythromycin, clindamycin, trimethoprim-sulfamethoxazole (except for 6 isolates), gentamicin and ciprofloxacin. Also, 24 isolates in this study were multidrug-resistant and methicillin susceptible S.aureus (MDR-MSSA). All these isolates were resistant to amoxicillin, erythromycin and tetracycline, and the majority was resistant to ciprofloxacin (79\%) and clindamycin $(62.5 \%)$. The majority of MDR isolates were belonged to ICU setting. The resistance to trimethoprim- sulfamethoxazole and gentamicin between MDR-MRSA and MDR- MSSA were significant (table2). All the MDR-MRSA and the majority of MDR- MSSA isolates were strong biofilm producers in the Mtp assay.

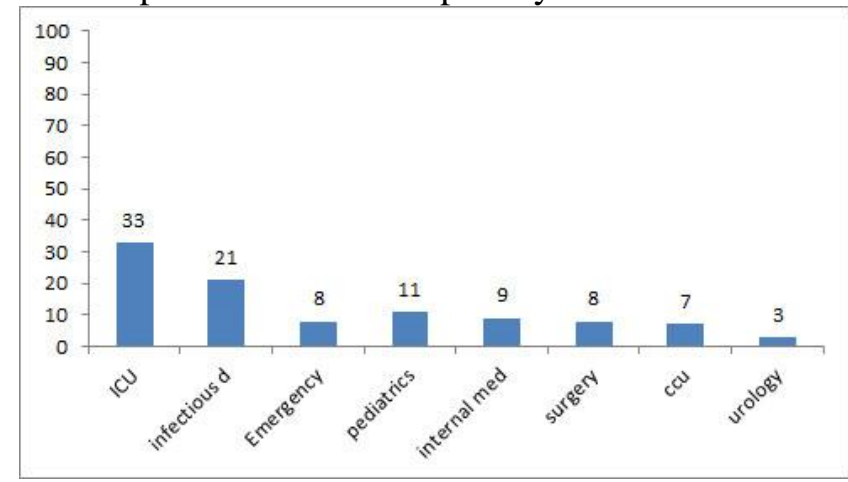

Figure 1: The hospital settings from which the bacterial isolates were collected

All the MDR-MRSA carried SCCmec type III with a 280 bp size PCR product. The rate of antibiotic resistance among these isolates was significantly higher than MSSA $(P v=0.012)$. Six isolates were susceptible to co- trimoxazole (SXT). In the current study, isolates with SCCmec type III were resistant to more variety of antibiotics compared to other SCC types. The majority of MDR- MRSA was belonged to $\operatorname{agrI}(67 \%, \mathrm{n}=24)$, followed by agr II $(17 \%, \mathrm{n}=6)$, $\operatorname{agrIV}(11 \%, \mathrm{n}=4)$ and $\operatorname{agrIII}(5.5 \%, \mathrm{n}=2)$. There was not a confirmed relationship between the $a g r$ groups and antibiotic susceptibility pattern or biofilm production by the isolates.

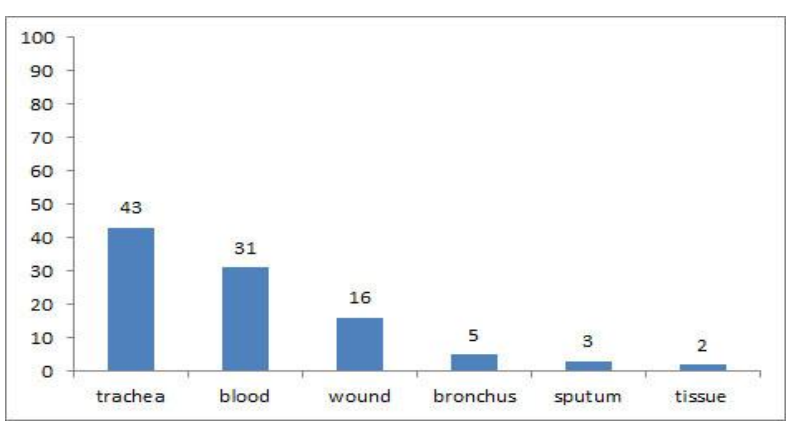

Figure 2: Different clinical sites the bacterial isolates
The frequency of icaADBC genes in MDR-MRSA were $75 \%(\mathrm{n}=27), 61 \%(\mathrm{n}=22), 72 \%(\mathrm{n}=26)$ and $72 \% \quad(\mathrm{n}=26)$, respectively. Furthermore, the prevalence of clfA, clfB, fnbA, fnbB, fib, can, eno, ebps and $b b p$ genes was $100 \%, 100 \%, 67 \%, 56 \%$, $80 \%, 78 \%, 7 \%$ and $0 \%$, respectively (table 2 ). No relation between each of the agr specific groups and biofilm encoding genes among these isolates was confirmed as exhibited in table 3. The high prevalence of these associated genes was correlated with strong biofilm production in the isolates. The differences among MSSA, MRSA and MDR isolates regarding biofilm formation and the presence of biofilm related genes has been depicted in tubular form 3 .

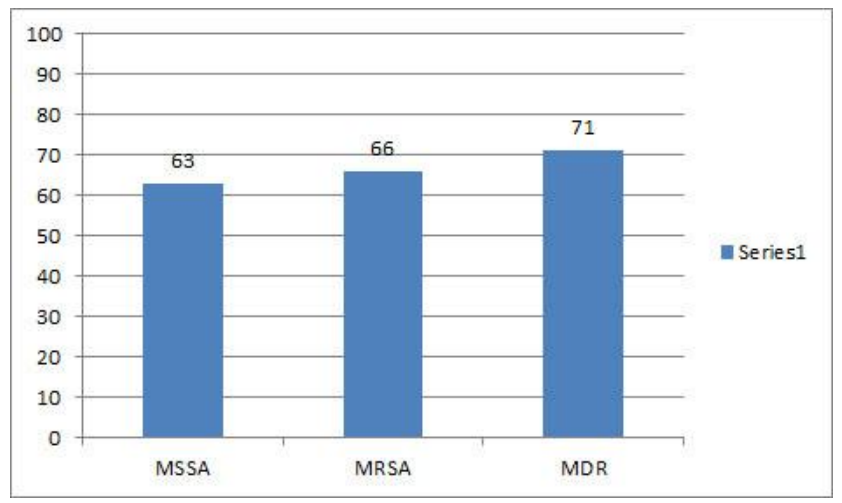

Figure 3: The differences among MSSA, MRSA and MDR isolates regarding biofilm formation and the presence of biofilm related genes

\section{Discussion}

In the present study, the prevalence of MRSA was $30.6 \%$. In previous studies it was not high ${ }^{14-16}$. However several other published surveys from Iran have determined that MRSA frequency is relatively high $^{14-15}$. Systemic review by Askari ${ }^{14}$ exhibited that that MRSA prevalence is more than $50.0 \%$ in Iran. The difference in these results originates mainly from differences in the genetic background of strains, clinical origins and epidemiological areas of studies. In this present study, the MRSA isolates exhibited significantly more resistance to the used antibiotics, except to vancomycin and linezolid. Among the 64 MRSA 36(56\%) isolates were resistant to the all the antibiotics contained SCCmec type III. Although 6 isolates with SCCmec type III were only susceptible to SXT, vancomycin and linezolid. Moreover, 24 MSSA isolates were MDR $S$. aureus. All these isolates were resistant to amoxicillin, tetracycline and erythromycin. The majority of MDR isolates were from ICU patients indicating that the isolates may be related to each other. In the phenotypic biofilm production, the MDR isolates produced biofilms 
strongly; which indicates that biofilm production can highly affect the extent of antibiotic resistance among multidrug-resistant isolates. Several previous studies have demonstrated this relationship ${ }^{9,16}$. In fact the SCCmec type III contains a variety of genetic elements that confer resistance to several antibiotics and other compounds. According to previous results the majority of healthcare associated MRSA harbor the SCCmec type III $^{15,17}$. Also these MRSA were significantly more resistant to antibiotics enhancing the idea that these isolates had caused hospital acquired infections. In this study, all the MRSA with other SCCmec types (I, V) showed resistance to a lower number of the antibiotics. This study depicts the importance of SCCmec type III in the multidrug antibiotic resistance of MRSA. The majority (73.2\%, $\mathrm{n}=26$ ) of multidrug MRSA was belonged to agrI $(67 \%, \mathrm{n}=24)$ followed by agrII $(19.5 \%, \mathrm{n}=7)$, agrIV $(8 \%, \mathrm{n}=3)$ and agrIII $(5.5 \%, \mathrm{n}=2) . \quad$ In previous studies agrI was the predominant specific group ${ }^{16}$. The agr groups play an important role in the regulation of several virulence factors of $S$. aureus. However, the relationships between each specific group and pathogenesis, clinical manifestations and drug resistance of MRSA isolates have not been certainly determined.

In this study, all the MDR- MRSA strains were capable of producing biofilm strongly by attachment into wells of micro-titre tissue plates. This result emphasizes the role of biofilm formation in various persistent and chronic infections caused by MDRMRSA that do not response to antimicrobial therapy. Furthermore, the majority (72\%) of multidrug MRSA harbored icaAD genes necessary for biofilm formation. As mentioned, these gene form biofilm via a synthesis of a Polysaccharide Inter-cellular Adhesion (PIA). The frequency of icaADBC genes were $75 \%(n=27), 61 \%(n=22), 72 \%(n=26)$ and $72 \%(n=26)$ respectively. The high prevalence of the ica genes along with strong biofilm formation justifies the resistance of MRSA isolates to a myriad of adverse conditions in addition to antibiotics. Furthermore, in the previous study, all these isolates can express the biofilm related genes in Real time PCR assay. PIA related biofilms are mainly acquired from medical devices and catheters and culminate in systemic infections, and also make the treatment very more difficult ${ }^{14-15}$. In this study several patients had died albeit antibiotic therapy. Previous studies have also determined the high prevalence and importance of these genes in biofilm producing isolates. Semczuk et $\mathrm{al}^{18}$ have mentioned that all the isolates producing biofilm phenotypically harbored icaAD genes. In Hou's study ${ }^{14}$, among $55.56 \%$ of isolates that produced biofilm in phenotypic test, $11.11 \%$ contained the icaA gene; however, the other genes have not been investigated. In this study, methicillin resistant isolates harbored higher rate of icaADBC genes compared to MSSA; no significant difference was confirmed, similar to two other studies ${ }^{15-16}$. Moreover, Smith ${ }^{17}$ determined no significant relation between susceptibility to methicillin and biofilm formation.

Likewise, Rasha ${ }^{19}$ detected the icaAD genes in $32 \%$ of blood and catheter isolates. In Zmantar's study ${ }^{20}$, 36 of 46 Staphylococcal isolates harbored icaA and icaD genes; while Grinholc and coworkers ${ }^{21}$ did not detect icaD but all strains were icaA positive ${ }^{14}$. Meriem detected the icaAD genes in $17(38.5 \%)$ of the 44 Staphylococcal isolates from urinary tract. In the Wang study ${ }^{20}$, biofilm formation in most of the isolates was PIA dependent. On the other hand, clinical origin of the isolates and infection site may be an important factor in the ability of the isolates to biofilm formation. Smith ${ }^{17}$ depicted that isolates of $S$. aureus from infected skin lesions were significantly more capable of producing biofilms than those isolated from blood and other infected sites. Elena ${ }^{21}$ suggests that the ica genes might be more prevalent in Staphylococcus strains isolated from the hospitalized patients or the staff, than healthy individuals or from the community. The previous studies about biofilm production in MDR-MRSA isolates are scarce. However, there is a relationship between these isolates and biofilm formation. Furthermore, in this study the prevalence of clfA, clfB, fnbA, fnbB, fib, eno, can, ebps and bbp genes was $100 \%, 100 \%, 67 \%, 56 \%, 80 \%, 78 \%, 65 \%, 7 \%$ and $0 \%$, respectively. Similarly, in Atshan's study ${ }^{22}$, all MRSA and MSSA strains harbored $c l f A, B$ genes. Investigation showed that $69.7 \%$ of the isolates harbored $c l f A B$ genes $^{22}$. However, Momtaz ${ }^{23}$ confirmed that nearly $20 \%$ of S. aureus isolates causing mastitis contained clfA gene. It seems that the kind of clinical isolates according to site of infections may be important in the prevalence of these genes essential for colonization in addition to the epidemiological differences. Furthermore, all the MRSA and MSSA isolates harbored $c l f A B$ genes. In this study the prevalence of $f n b A$ and $f n b B$ was $60 \%$ and $47 \%$, respectively. A study by Wastfelt ${ }^{24}$ has detected the $f i b$ gene in all $S$. aureus strains. Similarly, in previous study ${ }^{20}$ all isolates that conferred clindamycin inducible resistance and also vancomycin intermediate $S$. aureus (VISA) isolates carried this gene. However, in phenotypic test VISA isolates produced lower amount of biofilms. The prevalence of eno and cna genes was $78 \%$ and 63\%, respectively, showing the important role of these genes in colonization of $S$. aureus. Because of the high presence of laminin and collagen in tissues, $S$. aureus isolates can easily and rapidly bind to the 
specific receptors. The limitation of this study was low number of MDR isolates; thus more studies are needed in future to detect biofilm formation in MDR nosocomial isolates.

\section{Conclusion}

All the MRSA with multiple antibiotic resistances contained SCCmec type III and belonged to agrI. The frequency of different biofilm associated genes and likewise the amount of biofilm production was high in MDR-MRSA isolates. MDR isolates of S.aureus might be capable of strong biofilm formation that contributes to the higher antibiotic resistance.

\section{References}

1. Cespedes C, Miller M, Quagliarello B, Vavagiakis P, Klein RS, Lowy FD. Differences between Staphylococcus aureus isolates from medical and nonmedical hospital personnel. Journal of clinical microbiology 2002; 40(7): 2594-2597

2. Nickerson EK, West TE, Day NP, Peacock SJ. Staphylococcus aureus disease and drug resistance in resource-limited countries in south and east Asia. The Lancet infectious diseases 2009; 9(2): 130-135

3. Patel R. Biofilms and antimicrobial resistance. Clinical orthopaedics and related research 2005; 437: 41-47

4. Azimian A, Havaei SA, Fazeli $\mathrm{H}$ et al. Genetic characterization of a vancomycin-resistant Staphylococcus aureus isolate from the respiratory tract of a patient in a university hospital in northeastern Iran. Journal of clinical microbiology 2012; 50(11): 3581-3585

5. Kinnevey PM, Shore AC, Brennan GI et al. Emergence of sequence type 779 methicillin-resistant Staphylococcus aureus harboring a novel pseudo staphylococcal cassette chromosome mec (SCCmec)-SCC-SCCCRISPR composite element in Irish hospitals. Antimicrobial agents and chemotherapy 2013; 57(1): 524-531

6. Mah T-F. Biofilm-specific antibiotic resistance. Future microbiology 2012; 7(9): 1061-1072

7. Hoiby N, Bjarnsholt T, Givskov M, Molin Sr, Ciofu O. Antibiotic resistance of bacterial biofilms. International journal of antimicrobial agents 2010; 35(4): 322-332.

8. Anderson GG, O'Toole GA. Innate and induced resistance mechanisms of bacterial biofilms. Bacterial Biofilms: Springer, 2008: 85-105

9. Kwon AS, Park GC, Ryu SY et al. Higher biofilm formation in multidrug-resistant clinical isolates of Staphylococcus aureus. International journal of antimicrobial agents 2008; 32(1): 68-72

10. Pozzi C, Waters EM, Rudkin JK et al. Methicillin resistance alters the biofilm phenotype and attenuates virulence in Staphylococcus aureus device-associated infections. PLoS pathogens 2012; 8(4): e1002626
11. Sanchez CJ, Mende K, Beckius ML et al. Biofilm formation by clinical isolates and the implications in chronic infections. BMC infectious diseases 2013; 13(1): 47.

12. Gey A, Werckenthin C, Poppert S, and Straubinger RK. Identification of pathogens in mastitis milk samples with fluorescent in situ hybridization. Journal of Veterinary Diagnostic Investigation 2013; 25(3): 386-394

13. Zhang K, McClure J-A, Elsayed S, Conly JM. Novel staphylococcal cassette chromosome mec type, tentatively designated type VIII, harboring class A mec and type 4 ccr gene complexes in a Canadian epidemic strain of methicillin-resistant Staphylococcus aureus. Antimicrobial agents and chemotherapy 2009; 53(2): 531-540

14. Askari E, Soleymani F, Arianpoor A, Tabatabai SM, Amini A, NaderiNasab M. Epidemiology of mecA-methicillin resistant Staphylococcus aureus (MRSA) in Iran: A systematic review and meta-analysis. Iranian journal of basic medical sciences 2012; 15(5):1010

15. Mirzaee M, Najar Peerayeh S, Ghasemian A-M. Detection of icaABCD Genes and Biofilm Formation in Clinical Isolates of Methicillin Resistant Staphylococcus aureus. Iranian Journal of Pathology 2014; 9(4): 257-262

16. Gill SR, Fouts DE, Archer GL et al. Insights on evolution of virulence and resistance from the complete genome analysis of an early methicillin-resistant Staphylococcus aureus strain and a biofilm-producing methicillin-resistant Staphylococcus epidermidis strain. Journal of bacteriology 2005; 187(7): 2426-2438

17. Ghasemian A, Peerayeh SN, Bakhshi B, Mirzaee M. Detection of accessory gene regulator groups genes and cassette chromosome mec types among Staphylococcus aureus isolated from intensive care unit patients. Asian Pacific Journal of Tropical Disease 2015; 5(2): 153-157

18. Semczuk K, Dzierzanowska-Fangrat $K$, Dmeaska $H$, Dzierzanowska D. The analyze of capability of biofilm synthesis by Staphylococcus aureus strains isolated from children with cystic fibrosis. Medycyna doswiadczalna i mikrobiologia 2007; 60(4): 311-318

19. Nasr RA, AbuShady HM, Hussein HS. Biofilm formation and presence of icaAD gene in clinical isolates of staphylococci. Egyptian Journal of Medical Human Genetics 2012; 13(3): 269-274

20. Zmantar T, Kouidhi B, Miladi H, Mahdouani K, Bakhrouf A. A microtiter plate assay for Staphylococcus aureus biofilm quantification at various $\mathrm{pH}$ levels and hydrogen peroxide supplementation. The new microbiologica 2010; 33(2): 137

21. Grinholc M, Wegrzyn G, Kurlenda J. Evaluation of biofilm production and prevalence of the icaD gene in methicillinâ€ $\square$ resistant and methicillinâ€ $\square$ susceptible Staphylococcus aureus strains isolated from patients with nosocomial infections and carriers. FEMS Immunology \& Medical Microbiology 2007; 50(3): 375-379

22. Miller LG, Perdreau-Remington F, Rieg G et al. Necrotizing fasciitis caused by community-associated methicillinresistant Staphylococcus aureus in Los Angeles. New England Journal of Medicine 2005; 352(14): 1445-1453. 